\title{
Comparison of Efavirenz Release in Biorelevant Dissolution Media Using Manual Sampling and In Situ UV Fiber Optic System
}

\author{
Thalita Martins da Silva ${ }^{1,2,3}$, Marcelo Henrique da Cunha Chaves ${ }^{2,3}$ Beatriz Ferreira de Carvalho \\ Patricio ${ }^{2,3}$, and Helvécio Vinícius Antunes Rocha1,2,3* \\ ${ }_{1}^{1}$ Postgraduate Program in Management, Research and Development in the Pharmaceutical Industry, Oswaldo Cruz Foundation, Rio de Janeiro, Brazil. \\ ${ }^{2}$ Laboratory of Micro and Nanotechnology, Institute of Technology of Drugs, Oswaldo Cruz Foundation, Rio de Janeiro, Brazil. \\ ${ }^{3}$ Rio de Janeiro Innovation Netword in Nanosystems for Health - NanoSaúde/ FAPERJ, Rio de Janeiro, Brazil.
}

e-mail: helvecio.far@gmail.com

\begin{abstract}
There has been a growing interest in the use of biorelevant dissolution media in drug formulations research and development. Biorelevant media mimic the physiological conditions of the human gastrointestinal tract, which allows for a more discriminating dissolution test. That is even more important for poorly soluble drugs, like efavirenz and other class II (Biopharmaceutical Classification System) drugs. Traditionally, these media were used in the compendial standard dissolution apparatus; however, reduced automation of the conventional tests could be, in general, costly, high-labor, and error-prone. Thus, the use of in situ ultraviolet (UV) fiber optic probes has been applied in research and development and in quality control routines for drug manufacturing. The present study aims to assess the suitability of an in situ UV dynamic monitoring system for characterization of dissolution behavior of 600-mg efavirenz immediate-release coated tablets in different biorelevant media by comparison with traditional manual sampling. The biorelevant media used were fasted and fed state simulated intestinal fluid (FaSSIF, FaSSIF-V2, FeSSIF, FeSSIF-V2) and SLS (0.5\%). Both sampling methods were similar for FaSSIF, FaSSIF-V2, and SLS. For FeSSIF and FeSSIF-V2, the results were statistically different due to high concentration of oxidation phospholipids and degradation lipids in these media. These results support the use of dynamic monitoring of dissolution in FaSSIF, FaSSIF-V2, and SLS, and inform understanding of the rate-limiting steps, which may improve quality and accuracy in data acquisition for dissolution tests with biorelevant media.
\end{abstract}

KEYWORDS: UV fiber optics, in situ measurement, fiber optic dissolution system, biorelevant dissolution media, BCS class II, efavirenz, dissolution

\section{INTRODUCTION}

deally, a dissolution medium should expose the oral pharmaceutical dosage to the same challenges as it will face in the gastrointestinal (GI) tract, which involves changes in $\mathrm{pH}$, transit time, and buffering capacity, as well as the whole digestion physiology (1). In general, compendial dissolution media include acid solutions, buffering systems, or synthetic surfactants to reproduce the in vivo dissolution behavior. In some cases, these media could be far from the conditions that the pharmaceutical dosage form will face, especially for poorly soluble drugs, i.e., class II in the Biopharmaceutical Classification System (BCS) (2). Buffering systems are not physiologically relevant enough to achieve sink conditions through a practical volume of dissolution medium. To overcome this problem, it is common to use synthetic surfactants that allow sink conditions but could overestimate the in vivo dissolution rate (3). As an alternative, it is of great interest to the scientific community and drug manufacturers to use biorelevant dissolution media that mimic the GI tract physiological conditions and, in most cases, use surfactants that are present in human intestinal fluids such as sodium taurocholate and phosphatidylcholine to simulate bile salts and phospholipids, respectively (4). These media can be adjusted for $\mathrm{pH}$, osmolarity, superficial tension, and other physicochemical properties to simulate conditions in specific segments of the GI tract, which allows a drug solubility enhancement $(5,6)$.

Efavirenz (EFV) is an antiviral BCS class II drug, approved for acquired immunodeficiency syndrome (AIDS) 
treatment against human immunodeficiency virus (HIV) type 1. It is also considered a brick dust drug owing to its limited dissolution and low bioavailability (7). In this way, a dissolution test with biorelevant media could discriminate factors that contribute to the in vivo drug behavior (8).

Galia et al. proposed the first generation of intestinal simulation fluid, and it became known as fasted and fed state simulated intestinal fluid (FaSSIF and FeSSIF, respectively) (9). The next generation, based on physiological bile salt and phospholipids studies, was proposed by Jantratid et al. and became known as version 2 (i.e., FaSSIF-V2 and FeSSIF-V2, respectively) (10). The newest versions use maleate buffer instead of phosphate and acetate, which better reflects in vivo conditions by preventing early oxidation of fatty acids. Also, the FeSSIF-V2 presents a lipidic degradation product as oleate and glycerol monooleate, which could promote a dissolution enhancement and better represent the fed state (10).

The review presented by Bou-Chacra et al. points out that, in the last decade, the use of biorelevant dissolution media to evaluate drug solubility and dissolution has increased, especially for BCS class II drugs (6). Dissolution methods using biorelevant media are reported as a reliable and reproducible for the analysis of drug candidates and dosage form development. Also, it provides a way to establish more predictive dissolution methods for the in vivo process (11).

Traditionally, the drug release profile is measured through manual sampling, processing, and quantification by classical methods, such as ultraviolet (UV) spectrophotometry (12). However, manual sampling during the dissolution studies leads to an increase in study time and variability of the results, which may make it difficult to discriminate formulation changes (13). The most common sampling errors involve incorrect sampling times, sampler position, filters, or handling (14).

The in situ dissolution quantification technique was introduced in 1988 (15). These systems share most of the elements of traditional spectrophotometers. Though, instead of using a cuvette for sample storage, optical fibers transmit UV light from the source to the sample and the detector. The optical path is defined by the in situ probes. The systems diverge, mostly, with respect to the probe design and the detector type (16).

The drug release dynamic monitoring system could also be applied in quality control routines because the technique yields complete profiles and real-time results, thereby informing out of specification tests (17). Moreover, the in situ system is less labor-intensive and less prone to error (18). The method expanded its relevance due to its application in quality control and validation capacities $(13$, 19). The United States Pharmacopoeia (USP) identified this detection method as an alternative dissolution test that could be validated, with advantages in terms of precision, sensibility, selectivity, automation (20).

The use of this dynamic system is also found in predictive dissolution studies, as it allows for drug characterization and provides information about the initial minutes of the dissolution test (18). However, there are few data in the literature relating biorelevant dissolution media and the in situ UV probe quantification method. Li et al. described a $\mathrm{pH}$-gradient dissolution method combined with permeability measures to assess bioavailability reduction of poorly soluble compounds due to drugdrug interactions (21). The system was able to accurately measure the permeability changes according to the tested formulation, and it was possible to correlate their results with data reported in clinical studies. Other studies have used the dynamic monitoring system with biorelevant media through a micro-dissolution apparatus $(22,23)$.

Given the complex composition of biorelevant dissolution media, drug release quantification in these conditions could be a challenge. Therefore, this study aims to assess the suitability of the in situ dissolution dynamic monitoring system to measure the release profile of EFV tablets in different biorelevant dissolution media and SLS $(0.5 \%)$ by comparison with data obtained from traditional manual sampling.

\section{MATERIALS AND METHODS}

\section{Materials}

All solvents used in this study were of chromatographic grade, and all reagents used were of analytic grade. Materials included sodium lauryl sulfate (SLS) (BASF, Germany), sodium chloride, hydrochloride acid, glacial acetic acid (Merck, Germany), maleic acid, sodium hydroxide (Sigma-Aldrich, USA), sodium dihydrogen phosphate (Spectrum, Canada), FaSSIF, FeSSIF, FaSSIF-V2, and FeSSIF-V2 (all from Biorelevant, London, UK). Farmanguinhos/Fiocruz (Brazil) provided 600-mg EFV immediate-release coated tablets (lot 180150151). The manufacturer of the active pharmaceutical ingredient (API) cannot be disclosed due to a confidentiality agreement.

\section{Preparation of Dissolution Media}

The dissolution media were prepared as recommended by the manufacturer (24). The desired biorelevant

Dissolution r 
medium was prepared by dissolving the appropriate amount of powder in the recommended blank buffer. For FaSSIF, $2.24 \mathrm{~g}$ of FaSSIF powder was added to 1.0 $\mathrm{L}$ of phosphate buffer (pH 6.5). For FeSSIF, $11.20 \mathrm{~g}$ of FeSSIF powder was added to $1.0 \mathrm{~L}$ of acetate buffer $(\mathrm{pH}$ 5.0). Both FaSSIF and FeSSIF solutions were kept at rest for 4 hours before use. For FaSSIF-V2, $1.79 \mathrm{~g}$ of powder was added to $1.0 \mathrm{~L}$ of maleate buffer (pH 6.5). Finally, for FeSSIF-V2, $9.76 \mathrm{~g}$ of powder was added to $1.0 \mathrm{~L}$ of maleate buffer ( $\mathrm{pH}$ 5.8). Both V2 solutions were kept at rest for 1 hour before use, as recommended by the manufacturer. To prepare SLS $(0.5 \% \mathrm{w} / \mathrm{v}), 5.00 \mathrm{~g}$ of SLS was added to 1.0 $L$ of purified water. All dissolution media were vacuum degassed before use.

\section{Dissolution Test Using In Situ Method}

For the in situ method, dissolution of EFV tablets was evaluated in triplicate using a USP apparatus 2 (Evolution 6100, Distek Inc., NJ, USA). The drug concentration in each vessel was monitored online. The dissolution system uses a multi-channel in situ UV fiber-optic probe (Opt-Diss 410, Distek Inc.) with a path length of $10 \mathrm{~mm}$, exposure time of $129 \mathrm{~ms}$ (5 scans/data point), at an analytical wavelength of $293 \mathrm{~nm}$; baseline correction on an average wavelength was set at 350-400 nm. The whole UV spectrum of EFV API was obtained to confirm the use of the analytical wavelength. The blank intensity spectra were acquired for both standard solutions, dissolution media, and placebo suspension. The EFV amount was calculated based on EFV API standard solution by the Opt-Diss 410 MCA software (version 2.0.0, Distek Inc.).

The dissolution tests were conducted at $37 \pm 0.5^{\circ} \mathrm{C}$ in 900 $\mathrm{mL}$ dissolution media for SLS 0.5\%, FeSSIF, and FeSSIF-V2, and in $500 \mathrm{~mL}$ for FaSSIF and FaSSIF-V2, as reported by previous studies, at $50 \mathrm{rpm}$ for 150 minutes $(8,9,11)$.

\section{Dissolution Test Using Manual Sampling}

For the traditional manual sampling method, a similar experiment was conducted to compare the dissolution profile with the in situ method. The tests were not run simultaneously to avoid technical interferences during the assay, such as the reduction in dissolution media volume. The same volume of media, temperature, and agitation were used. Samples $(5 \mathrm{~mL})$ were filtered in a 45- $\mu \mathrm{m}$ polyethylene filter (Filtrilo, Paraná, Brazil) at 5,10 , $15,30,45,60,90,120$, and 150 minutes without media replacement. Before EFV quantification, samples were filtered again in a $0.45-\mu \mathrm{m}$ polyvinylidene difluoride membrane filter (Filtrilo) and analyzed offline using an ultra-high-performance liquid chromatography (UHPLC)
Acquity Arc (Waters, Massachusetts, USA). The EFV amount was calculated based on a calibration curve. These steps were described previously by Silva (25).

\section{Statistics}

The drug release profiles of each dissolution media were compared using the similarity factor $\left(f_{2}\right)$ as described in United States Food and Drug Administration (FDA) guidance for dissolution testing (26). Dissolution efficiency (DE) was used for comparison of dissolution rates and calculated by the area under the curve (AUC) through trapezoids methods. The DE was used to measure the influence of the quantification method. The $f_{2}$ calculations were conducted at the limit intervals to the dissolution plateau. When a plateau was not achieved, or the dissolution did not reach $100 \%$, a total of $85 \%$ of drug release was used. Data are calculated using DDSolver (version 1.0, China Pharmaceutical University, Nanjing, China) add-in program for Microsoft Excel (Office 365) (27).

Additionally, one- and two-way ANOVA post hoc Tukey tests were used to compare the DE and dissolution profiles, respectively. For quantification method comparison, a two-way ANOVA post hoc Sidak correction was used. In all cases, $p<0.05$ was considered to be statistically significant. Data were calculated using Action Stat (version 3.6.331.450, Estatcamp, São Paulo, Brazil) in Microsoft Excel (Office 365).

\section{RESULTS AND DISCUSSION}

In the present work, two identical dissolution tests were designed but performed with different quantification methods analyses (in situ UV fiber optics probe and manual sampling) to evaluate the influence of high complexity biorelevant media compounds in EFV dissolution and UV fiber optic probe quantification. The full UV spectrum of EFV API is presented in Figure 1 to justify the in situ monitoring using $293 \mathrm{~nm}$ wavelength. Efavirenz presents high absorbance intensity in $248 \mathrm{~nm}$, but in this wavelength the absorption intensity was too high, leading to a pronounced spread, mainly in FaSSIF-V2. Therefore, the wavelength of $293 \mathrm{~nm}$ was chosen for the evaluations in this study.

Both profiles presented a coefficient of variation under $20 \%$ on early time points ( $\leq 15$ minutes), and no more than $10 \%$ on the others (data not shown), per regulatory recommendations (26). Although the two tests in this study were not performed simultaneously, the results showed no relevant statistical differences from run to 
run. The differences in EFV release between the two versions of FaSSIF and FeSSIF and the comparison with the validated method using SLS $0.5 \%$ were extensively discussed previously by Silva (25).

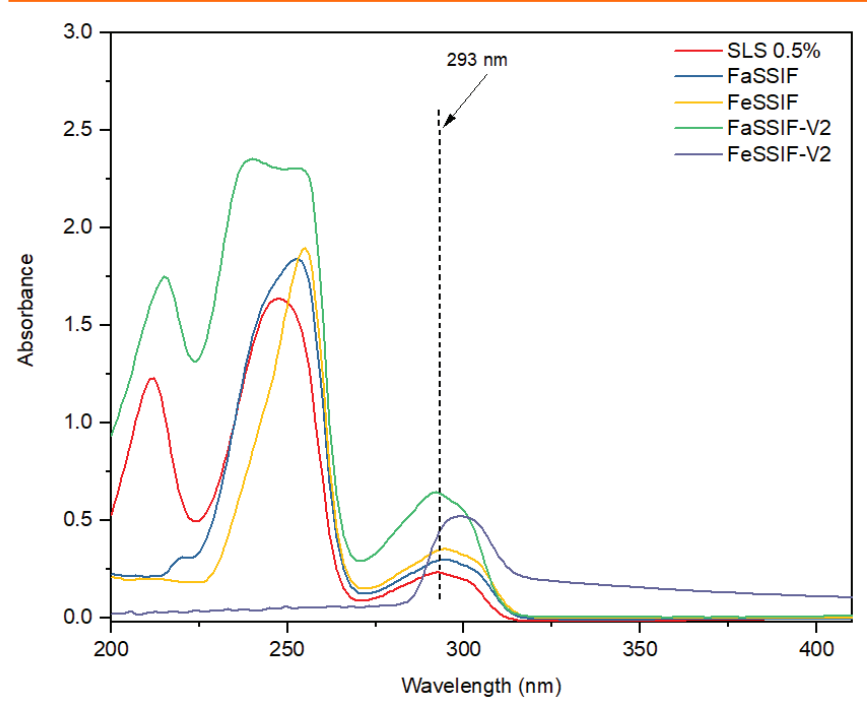

Figure 1. UV spectrum of efavirenz $(0.06 \mathrm{mg} / \mathrm{mL}) \mathrm{API}$ in different biorelevant media and SLS 0.5\% at $10 \mathrm{~mm}$ path length. SLS: sodium lauryl sulphate; FaSSIF: fasted state simulated intestinal fluid; FeSSIF: fed state simulated intestinal fluid; V2: version 2; API: active pharmaceutical ingredient.

Regarding the dissolution media, the profiles comparison by $f_{2}$ showed that the pairs: FaSSIF and FaSSIF-V2; FaSSIF and FeSSIF-V2; FaSSIF-V2 and FeSSIF-V2 had similar profiles (Table 1), because $f_{2}$ values were higher than 50 (26). However, when comparing the dissolution profiles by two-way ANOVA post hoc Tukey, the only pair that did not present a statistically significant difference was FeSSIF-V2 and FaSSIF (data not shown). As related by Yuksel et al. the ANOVA-based and model-dependent methods are more discriminative than $f$ factors. In the present study, $f_{2}$ was used only as a comparison criterion since this is the most compendium-recommended method (28).

Table 1. Efavirenz Tablet Dissolution Profile Comparison by Similarity Factor $\left(f_{2}\right)$ Using In Situ UV Probe

\begin{tabular}{|c|c|c|c|c|c|}
\hline $\begin{array}{c}\text { Dissolution } \\
\text { Media }\end{array}$ & SLS 0.5\% & FaSSIF & FeSSIF & FaSSIF-V2 & FeSSIF-V2 \\
\hline SLS 0.5\% & & 11.81 & 22.37 & 8.58 & 11.30 \\
\hline FaSSIF & 11.81 & & 31.73 & $\mathbf{5 1 . 2 8}$ & $\mathbf{8 5 . 6 0}$ \\
\hline FeSSIF & 22.37 & 31.73 & & 24.49 & 30.40 \\
\hline FaSSIF-V2 & 8.58 & $\mathbf{5 1 . 2 8}$ & 24.49 & & $\mathbf{5 4 . 3 1}$ \\
\hline FeSSIF-V2 & 11.30 & $\mathbf{8 5 . 6 0}$ & 30.40 & $\mathbf{5 4 . 3 1}$ & \\
\hline
\end{tabular}

Note - Data are $f_{2}$ values; boldface values are considered to be similar $\left(f_{2}>\right.$ 50). Results for manual sampling are included in (25).

UV: ultraviolet; SLS: sodium lauryl sulphate; FaSSIF: fasted state simulated intestinal fluid; FeSSIF: fed state simulated intestinal fluid; V2: version 2.
It is important to note that FeSSIF and FeSSIF-V2 simulate the fed state of the human GI tract, while FaSSIF and FaSSIF-V2 simulate the fasted condition. The statistical similarity $\left(f_{2}\right.$ and ANOVA) of the EFV release profile in biorelevant media with such different media (FeSSIF-V2 and FaSSIF) could be justified by the presence of the maleate buffer in FeSSIF-V2. According to the literature, this one has chromophores that could interfere in UV quantifications (5). Such interference might also be one explanation for statistical differences in EFV release in similar biorelevant media (i.e., FeSSIF and FeSSIF-V2).

Another reason to explain these differences is that, as shown in Figure 2, at the end of all dissolution tests, the media presented a high quantity of suspended particles, especially in FaSSIF and FaSSIF-V2. It probably occurred due to the reduced volume used $(500 \mathrm{~mL})$, which was recommended for simulation of such physiological conditions (29). The particulate matter could diffract the UV light, interfering with in situ quantification. As reported by Guillot et al., in the case of turbid suspensions, the API could present a too weak UV absorption in comparison with the total absorption of the suspension in the vessel. Turbid suspensions are one of the main limitations of fiber-optic systems use (30).
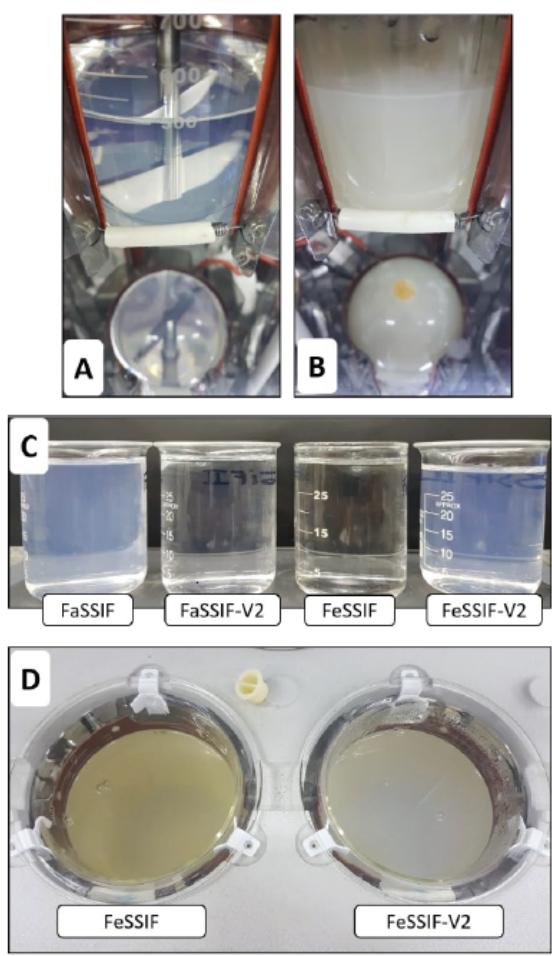

Figure 2. Biorelevant media before and after testing with an efavirenzcoated tablet, revealing a high number of suspended particles at the end of the dissolution test. A: FaSSIF before test; B: FaSSIF after 150 min of test; C: media used (before tests); D: FeSSIF and FeSSIF-V2 after 150 min of test. FaSSIF: fasted state simulated intestinal fluid; FeSSIF: fed state simulated intestinal fluid; V2: version 2. 
In comparison with the dissolution test conducted with manual sampling, as shown in Figure 3, the release profile using in situ UV probe presented no statistically significant differences in drug release in SLS 0.5\% (97.99\% for manual sampling vs $91.01 \%$ for UV probe), FaSSIF ( $15.73 \%$ vs $16.67 \%$ ) and FaSSIF-V2 (4.35\% vs 5.17\%) (25). On the other hand, statistically significant differences were recorded for FeSSIF ( $85.06 \%$ vs $55.68 \%, p<0.001)$ and FeSSIF-V2 (89.41\% vs $14.07 \%, p<0.001)$.

Concerning the quantification method, the test conducted with manual sampling shows that for SLS 0.5\% $\left(f_{2}=61.92\right)$, FaSSIF $\left(f_{2}=90.79\right)$, and FaSSIF-V2 $\left(f_{2}=97.79\right)$, the release profiles were similar in comparison with the in situ UV fiber optic system. However, FeSSIF and FeSSIF-V2 presented $f_{2}$ values of 40.06 and 17.18 , respectively. In the same way, the analysis by two-way ANOVA post hoc Tukey revealed that the use of in situ UV fiber optic was effective for EFV release evaluation in SLS 0.5\%, FaSSIF, and FaSSIF-V2 ( $p>0.05)$. Regarding the dissolution media, there was no statistical difference between the release profiles using in situ UV probe and manual sampling, as shown in Table 2.

Relating to quantification methods evaluation, Table 3 shows the DE values for the UV fiber optic and manual samples methods. FaSSIF, FaSSIF-V2, and SLS 0.5\% showed similar DE independently of the method of quantification $(p>0.05)$, indicating that the in situ dynamic monitoring of dissolution profile was appropriate for EFV in these media. For FeSSIF and FeSSIF-V2, the DE values were statistically different $(p<0.05)$; however, DE for FaSSIF and FeSSIF-V2 were similar with the UV fiber optic probe, and FeSSIF and FeSSIF-V2 were similar with manual sampling $(p>0.05)$.

As shown in Table 2, for FeSSIF and FeSSIF-V2, the influence of the quantification method was statistically significant $(p=0.003$ and $<0.001$, respectively), as

Table 2. Influence of Quantification Method on Efavirenz Tablet Dissolution Rate

\begin{tabular}{|c|c|}
\hline Dissolution Media & $\boldsymbol{p}$ \\
\hline SLS 0.5\% & 0.205 \\
\hline FaSSIF & 0.089 \\
\hline FaSSIF-V2 & 0.073 \\
\hline FeSSIF & $\mathbf{0 . 0 0 3}$ \\
\hline FeSSIF-V2 & $<\mathbf{0 . 0 0 1}$ \\
\hline
\end{tabular}

Note - Two-way analysis of variance was used for statistical comparison. Boldface values are statistically significant.

SLS: sodium lauryl sulphate; FaSSIF: fasted state simulated intestinal fluid; FeSSIF: fed state simulated intestinal fluid; V2: version 2. evidenced by differences in the amount of EFV dissolved, $\mathrm{DE}$, and dissolution profile when the quantification methods were compared.

Through the comparison of dissolution profiles, it is possible to conclude that, for EFV dissolution using in situ quantification, the maleate buffering system did not interfere, since the release profiles in FaSSIF and FaSSIF-V2 were similar. On the other hand, the phospholipid concentration could be the reason for the differences in EFV quantification in FeSSIF and FeSSIF-V2 with the in situ UV fiber optic system. According to Kloefer et al., FeSSIF has a high absorbance below $300 \mathrm{~nm}$ due to the phospholipid oxidation (31). Such interference is lower in FaSSIF due to the decrease in phosphatidylcholine concentrations. Despite the reduced phospholipid concentration in FeSSIF-V2, the lipid degradation products also interfered in the in situ quantification.

Based on the cost and technical complexity of biorelevant dissolution media, it is not expected to replace the evaluation performed with SLS in quality control routines, which is proven to be a predictive method for EFV tablets (32). The main objective of this study was to challenge the in situ quantification with the complex composition of biorelevant dissolution media, because there are no reported data for EFV in these conditions.

The main challenge of the in situ UV probe is performing drug quantification dynamically, without any sample treatment or filtering, where excipients and other complex molecules could absorb or scatter the UV light. To achieve this goal, the system uses mathematical filters that include a baseline correction and a second derivative calculation (18). In the present study, these filters were enough to evaluate the release profiles of EFV tablets in SLS, FaSSIF, and FaSSIF-V2. The use of these media was extensive to obtain a predictive dissolution profile

Table 3. Efavirenz Tablet Dissolution Efficiency (DE\%) Comparison by Quantification Method

\begin{tabular}{|c|c|c|c|}
\hline $\begin{array}{c}\text { Dissolution } \\
\text { Media }\end{array}$ & $\begin{array}{c}\text { In Situ UV Fiber } \\
\text { Optic Probe }\end{array}$ & $\begin{array}{c}\text { Manual } \\
\text { Sampling }\end{array}$ & p-Value \\
\hline SLS 0.5\% & $81.05( \pm 0.00)$ & $86.24( \pm 0.01)$ & 0.0554 \\
\hline FaSSIF & $\$ 15.41( \pm 0.00)$ & $14.24( \pm 0.00)$ & 0.9933 \\
\hline FaSSIF-V2 & $4.71( \pm 0.00)$ & $4.06( \pm 0.00)$ & 0.9994 \\
\hline FeSSIF & $45.51( \pm 0.03)$ & ${ }^{*} 61.15( \pm 0.03)$ & $<0.0001$ \\
\hline FeSSIF-V2 & $\$ 13.47( \pm 0.02)$ & ${ }^{*} 65.55( \pm 0.03)$ & $<0.0001$ \\
\hline
\end{tabular}

Note - Data are presented as mean DE\% $( \pm S D)(n=3)$.

${ }^{*}, \$$ - No significant statistical differences are present in the same quantification method.

SLS: sodium lauryl sulphate; FaSSIF: fasted state simulated intestinal fluid; FeSSIF: fed state simulated intestinal fluid; V2: version 2; SD: standard deviation. 

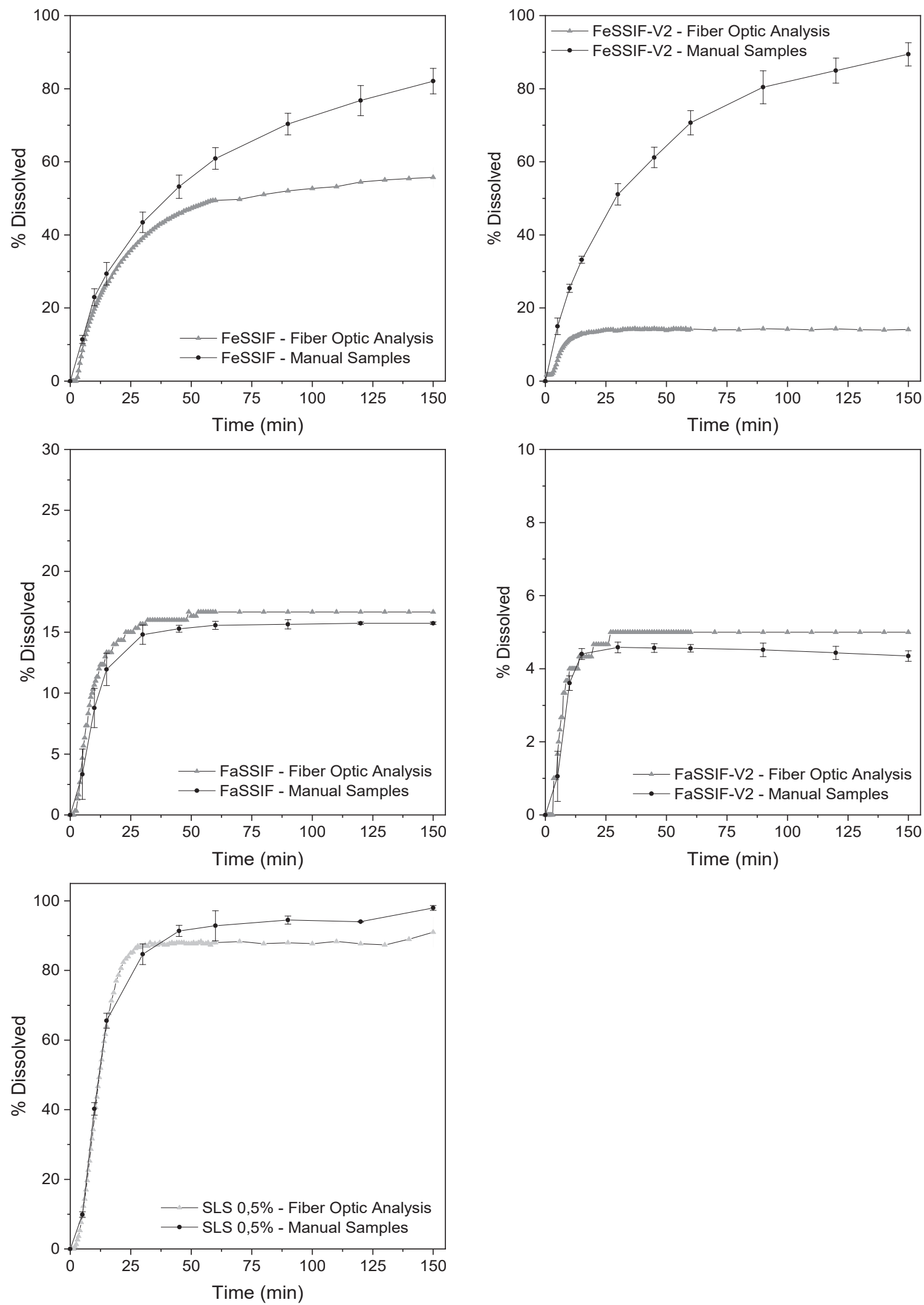

Figure 3. Dissolution profiles of efavirenz-coated tablets in biorelevant media (A-D) and SLS 0.5\% (E) measured by in situ fiber optic probe and manual sampling (data from 27). Data are presented as mean $\pm S D(n=3)$. SLS: sodium lauryl sulphate; FaSSIF: fasted state simulated intestinal fluid; FeSSIF: fed state simulated intestinal fluid; V2: version 2. 
that better reflects in vivo conditions. The fasted small intestinal conditions are the most common for assessing and comparing in vitro and in vivo data $(4,33,34)$.

The data evaluation using a second derivative baseline correction did not show remarkable results. In addition, a multicomponent analysis could also be performed, allowing the evaluation of different drugs or substances that absorb UV light in the same wavelength, as related by Nir and Lu (18). This correction may also allow for EFV monitoring in FeSSIF and FeSSIF-V2. Future analyses could be performed to overcome the UV absorption interference and establish a robust UV fiber optic method with these dissolution media.

\section{CONCLUSION}

The present work aimed to compare the dissolution profiles obtained by the traditional manual sampling and the in situ UV fiber optic probe for 600-mg EFV immediaterelease coated tablets. Through a statistical comparison between data obtained by the two techniques, results showed that the UV dynamic monitoring method is effective for biorelevant dissolution media, FaSSIF and FaSSIF-V2, although there was high suspension turbidity at the end of the tests and the presence of maleate buffer in the newer version. On the other hand, FeSSIF and FeSSIF-V2 presented different results when comparing dynamic monitoring and manual sampling. One possible reason is the presence of lipidic degradation products and the large amount of phosphatidylcholine in these media.

This work confirms that the fiber optic systems could be considered a suitable option for some dissolution media, facilitating in vitro drug release testing while reducing cost, time, human error, and labor. The dissolution test is especially important for poorly soluble drugs, like EFV, and the robust data provided by dynamic monitoring informs understanding of the rate-limiting steps. The dynamic dissolution monitoring technique with biorelevant media represents an increase in quality and accuracy with applications in research and development and quality control arenas.

\section{FUNDING}

This work was supported by Brazilian Development Bank (BNDES), National Council for Scientific and Technological Development (CNPQ), Carlos Chagas Filho Foundation for Research Support in the State of Rio de Janeiro (FAPERJ / E-26/010.000983/2019). The financing of all agencies was directed towards the acquisition of equipment and consumables. The objective is to create knowledge and critical knowledge regarding biorelevant dissolution tests that can be used as a predictive method of bioavailability and that have application for different drug products.

\section{CONFLICT OF INTEREST}

The authors disclosed no conflicts of interest related to this article.

\section{REFERENCES}

1. Long, M.; Chen, Y. Dissolution Testing of Solid Products. In Developing Solid Oral Dosage Forms: Pharmaceutical Theory and Pratice; Qiu, Y., Chen, Y., Zhang, G., Yu, L., Mantri, R. V., Eds.; Elsevier, 2009; pp 319-340. DOI: 10.1016/B978-0-444-532428.00014-X.

2. Bergström, C. A. S.; Box, K.; Holm, R.; Matthews, W.; McAllister, M.; Müllertz, A.; Rades, T.; Schäfer, K. J.; Teleki, A. Biorelevant intrinsic dissolution profiling in early drug development: fundamental, methodological, and industrial aspects. Eur. J. Pharm. Biopharm. 2019, 139, 101-114. DOI: 10.1016/j. ejpb.2019.03.011.

3. Löbenberg, R.; Amidon, G. L.; New Scientific Approaches to International Regulatory Standards. Modern bioavailability, bioequivalence and biopharmaceutics classification system. New scientific approaches to international regulatory standards. Eur. J. Pharm. Biopharm. 2000, 50, 3-12. DOI: 10.1016/S09396411(00)00091-6.

4. Dressman, J. B.; Amidon, G. L.; Reppas, C.; Shah, V. P. Dissolution testing as a prognostic tool for oral drug absorption: immediate release dosage forms. Pharm. Res. 1998, 15, 11-22. DOI: 10.1023/a:1011984216775.

5. Mauger, J. W. Physicochemical properties of buffers used in simulated biological fluids with potential application for in vitro dissolution testing: A mini-review. Dissolut. Technol. 2017, 24, 38-51. DOI: 10.14227/DT240317P38.

6. Bou-Chacra, N.; Melo, K. J. C.; Morales, I. A. C.; Stippler, E. S.; Kesisoglou, F.; Yazdanian, M.; Löbenberg, R. Evolution of choice of solubility and dissolution media after two decades of Biopharmaceutical Classification System. AAPS J. 2017, 19, 9891001. DOI: 10.1208/s12248-017-0085-5.

7. Cristofoletti, R.; Nair, A.; Abrahamsson, B.; Groot, D. W.; Kopp, S.; Langguth, P.; Polli, J. E.; Shah, V. P.; Dressman, J. B. Biowaiver monographs for immediate release solid oral dosage forms: Efavirenz. J. Pharm. Sci. 2013, 102, 318-329. DOI: 10.1002/ jps.23380.

8. Marques, M. R. C.; Loebenberg, R.; Almukainzi, M. Simulated biological fluids with possible application in dissolution testing. Dissolut. Technol. 2011, 18, 15-28. DOI: 10.14227/DT180311P15.

9. Galia, E.; Nicolaides, E.; Hörter, D.; Löbenberg, R.; Reppas, C.; Dressman, J. B. Evaluation of various dissolution media for predicting in vivo performance of class I and II drugs. Pharm. Res. 1998, 15, 698-705. DOI: 10.1023/A:1011910801212.

10. Jantratid, E.; Janssen, N.; Reppas, C.; Dressman, J. B. Dissolution media simulating conditions in the proximal human 
gastrointestinal tract: an update. Pharm. Res. 2008, 25, 16631676. DOI: 10.1007/s11095-008-9569-4.

11. Mann, J.; Dressman, J.; Rosenblatt, K.; Ashworth, L.; Muenster, U.; Frank, K.; Hutchins, P.; Williams, J.; Klumpp, L.; Wielockx, K.; Berben, P.; Augustijns, P.; Holm, R.; Hofmann, M.; Patel, S.; Beato, S.; Ojala, K.; Tomaszewska, I.; Bruel, J. L.; Butler, J. Validation of dissolution testing with biorelevant media: An OrBiTo study. Mol. Pharm. 2017, 14, 4192-4201. DOI: 10.1021/ acs.molpharmaceut.7b00198.

12. Provides for the conduct of pharmaceutical equivalence studies and comparative dissolution profile, RDC n. 31, Brazilian Health Regulatory Agency, Ministry of Health, August 11, 2010.

13. Liu, L.; Osei, T.; Hsu, J.; Greyling, J. Evaluation of in-situ fiber optics dissolution method for compound a extended release tablets. Am. Pharm. Rev. 2011, 14, 52-58.

14. Nir, I.; Johnson, B. D.; Johansson, J.; Schatz, C. Application of fibre optic dissolution testing for actual products. Pharm. Tech. 2001, 14: 33-40.

15. Josefson, M.; Johansson, E.; Torstensson, A. Optical fiber spectrometry in turbid solutions by multivariate calibration applied to tablet dissolution testing. Anal. Chem. 1988, 60, 2666-2671. DOI: 10.1021/ac00175a004.

16. Lu, X.; Lozano, R.; Shah, P. In-situ dissolution testing using different uv fiber optic probes and instruments. Dissolut. Technol. 2003, 10, 6-15. DOI: 10.14227/DT100403P6.

17. Gray, V. A. Dissolution testing using fiber optics - a regulatory perspective. Dissolut. Technol. 2003, 10, 33-36. DOI: 10.14227/ DT100403P33.

18. Nir, I.; Lu, X. In Situ UV Fiber Optics for Dissolution testing - what, why, and where we are after 30 Years. Dissolut. Technol. 2018, 25, 70-77. DOI: 10.14227/DT250318P70.

19. Mirza, T.; Liu, Q. J.; Vivilecchia, R.; Joshi, Y. Comprehensive validation scheme for in situ fiber optics dissolution method for pharmaceutical drug product testing. J. Pharm. Sci. 2009, 98, 1086-1094. DOI: 10.1002/jps.21481.

20. <1225> Validation of Compendial Procedures. The United States Pharmacopoeia and National Formulary, 42nd ed. United States Pharmacopial Convention, Inc.: Rockville, MD, 2019.

21. Li, J.; Tsinman, K.; Tsinman, O.; Wigman, L. Using pH gradient dissolution with in-situ flux measurement to evaluate bioavailability and DDI for formulated poorly soluble drug products. AAPS PharmSciTech 2018, 19, 2898-2907. DOI: 10.1208/s12249-018-1164-3.

22. Andersson, S. B. E.; Alvebratt, C.; Bevernage, J.; Bonneau, D.; da Costa Mathews, C.; Dattani, R.; Edueng, K.; He, Y.; Holm, R.; Madsen, C.; Müller, T.; Muenster, U.; Müllertz, A.; Ojala, K.; Rades, T.; Sieger, P.; Bergström, C. A. S. Interlaboratory validation of small-scale solubility and dissolution measurements of poorly water-soluble drugs. J. Pharm. Sci. 2016, 105, 2864-2872. DOI: 10.1016/j.xphs.2016.03.010.
23. Mathias, N. R.; Xu, Y.; Patel, D.; Grass, M.; Caldwell, B.; Jager, C.; Mullin, J.; Hansen, L.; Crison, J.; Saari, A.; Gesenberg, C.; Morrison, J.; Vig, B.; Raghavan, K. Assessing the risk of $\mathrm{pH}-$ dependent absorption for new molecular entities: A novel in vitro dissolution test, physicochemical analysis, and risk assessment strategy. Mol. Pharm. 2013, 10, 4063-4073. DOI: 10.1021/mp400426f.

24. Instructions, V1.1. Biorelevant.com: London, UK. 2017. Accessed July 13, 2021. https://biorelevant.com/site_media/upload/ documents/Biorelevant_Instructions_V1.1.pdf.

25. da Silva, T. M. [Development of a biorelevant dissolution method for efavirenz tablets and in vitro-in vivo correlation] [in Portuguese]. M.S. Dissertation, Oswaldo Cruz Foundation, Institute of Drug Technology, Rio de Janeiro, Brazil, 2019.

26. Dissolution Testing of Immediate Release Solid Oral Dosage Forms; Guidance for Industry; U.S. Department of Health and Human Services, Food and Drug Administration, Center for Drug Evaluation and Research (CDER), U.S. Government Printing Office: Washington, DC, 1997.

27. Zhang, Y.; Huo, M.; Zhou, J.; Zou, A.; Li, W.; Yao, C.; Xie, S. DDSolver: An add-in program for modeling and comparison of drug dissolution profiles. AAPS J. 2010, 12, 263-271. DOI: 10.1208/s12248-010-9185-1.

28. Yuksel, N.; Kanik, A. E.; Baykara, T. Comparison of in vitro dissolution profiles by ANOVA-based, model-dependent and -independent methods. Int. J. Pharm. 2000, 209, 57-67. DOI: 10.1016/S0378-5173(00)00554-8.

29. Marques, M. Dissolution media simulating fasted and fed states. Dissolut. Technol. 2004, 11, 16. DOI: 10.14227/DT110204P16.

30. Guillot, A.; Limberger, M.; Krämer, J.; Lehr, C. M. In situ drug release monitoring with a fiber-optic system: overcoming matrix interferences using derivative spectrophotometry. Dissolut. Technol. 2013, 20, 15-19. DOI: 10.14227/DT200213P15.

31. Kloefer, B.; van Hoogevest, P.; Moloney, R.; Kuentz, M.; Leigh, M. L. S.; Dressman, J. Study of a standardized taurocholate- lecithin powder for preparing the biorelevant media FeSSIF and FaSSIF. Dissolut. Technol. 2010, 17, 6-13.DOI: 10.14227/DT170310P6.

32. Honório, T. da S.; Pinto, E. C.; Rocha, H. V. A.; Esteves, V. S. D.; dos Santos, T. C.; Castro, H. C. R.; Rodrigues, C. R.; de Sousa, V. P.; Cabral, L. M. In vitro-in vivo correlation of efavirenz tablets using GastroPlus ${ }^{\circledR}$. AAPS PharmSciTech 2013, 14, 1244-1254. DOI: 10.1208/s12249-013-0016-4.

33. Grbic, S.; Parojcic, J.; Ibric, S.; Djuric, Z. In vitro-in vivo correlation for gliclazide immediate-release tablets based on mechanistic absorption simulation. AAPS PharmSciTech 2011, 12, 165-171. DOI: 10.1208/s12249-010-9573-y.

34. Schwebel, H. J.; van Hoogevest, P.; Leigh, M. L. S.; Kuentz, M. The apparent solubilizing capacity of simulated intestinal fluids for poorly water-soluble drugs. Pharm. Dev. Technol. 2011, 16, 278-286. DOI: 10.3109/10837451003664099. 